\title{
Botulinum toxin A inhibits salivary secretion of rabbit submandibular gland
}

\author{
Xiao-Feng Shan ${ }^{1}$, Hui Xu ${ }^{1}$, Zhi-Gang Cai ${ }^{1}$, Li-Ling $\mathrm{Wu}^{2}$ and Guang-Yan $\mathrm{Yu}^{1}$ \\ Botulinum toxin A (BTXA) has been used in several clinical trials to treat excessive glandular secretion; however, the precise \\ mechanism of its action on the secretory function of salivary gland has not been fully elucidated. In this study, we aimed to investigate \\ the effect of BTXA on secretion of submandibular gland in rabbits and to identify its mechanism of action on the secretory function of \\ salivary gland. At 12 weeks after injection with 5 units of BTXA, we found a significant decrease in the saliva flow from submandibular \\ glands, while the salivary amylase concentration increased. Morphological analysis revealed reduction in the size of acinar cells with \\ intracellular accumulation of secretory granules that coalesced to form a large ovoid structure. Expression of M3-muscarinic \\ acetylcholine receptor (M3 receptor) and aquaporin-5 (AQP5) mRNA decreased after BTXA treatment, and distribution of AQP5 in the \\ apical membrane was reduced at 1, 2 and 4 weeks after BTXA injection. Furthermore, BTXA injection was found to induce apoptosis of \\ acini. These results indicate that BTXA decreases the fluid secretion of submandibular glands and increases the concentration of \\ amylase in saliva. Decreased expression of M3 receptor and AQP5, inhibition of AQP5 translocation, and cell apoptosis might involve in \\ BTXA-reduced fluid secretion of submandibular glands. \\ International Journal of Oral Science (2013) 5, 217-223; doi:10.1038/ijos.2013.82; published online 25 October 2013
}

Keywords: aquaporin-5; apoptosis; botulinum toxin A; M3-muscarinic acetylcholine receptor; submandibular gland

\section{INTRODUCTION}

Botulinum toxin type A (BTXA) is a protein that has been used for the successful treatment of various neuromuscular and movement disorders. ${ }^{1-4}$ Recently, BTXA is used to treat the excessive glandular secretion such as hyperhidrosis, hyperlacrimation, allergic rhinitis and drooling, and the results were encouraging. ${ }^{5-7}$ However, most of the reports of BTXA-modulated secretion are from clinical trials. ${ }^{5-7}$ Morphological and immunohistochemical studies have revealed that intraglandular BTXA application in rat submandibular gland (SMG) leads to glandular atrophy and decreased expression of acetylcholine esterase. $^{8}$ The mechanism of decreased salivary secretion might be due to glandular atrophy and denervation induced by the inhibition in acetylcholine release at the neuroglandular junction. ${ }^{8}$

Salivary secretion is controlled by both sympathetic and parasympathetic autonomic nerves. Fluid and electrolyte secretion from salivary glands is primarily evoked by the action of acetylcholine on muscarinic receptors and norepinephrine on $\alpha$-adrenoceptors, whereas protein secretion is mainly evoked by isoproterenol on $\beta$-adrenoceptors. ${ }^{9-10}$ After the muscarinic cholinergic receptors are activated, the formation of inositol 1,4,5-trisphosphate and the increased intracellular calcium $\left(\mathrm{Ca}^{2+}\right)$ level could subsequently induce the activation of aquaporin-5 (AQP5), which in turn leads to transcellular secretion of water. ${ }^{11-12}$ It remains unknown whether BTXA decreases the secretion of salivary glands by inhibiting the function of muscarinic receptor signaling pathway. In this study, we aimed to investigate the effect of
BTXA on secretion of SMG in rabbits and to identify its molecular mechanism to regulate the secretory function of salivary glands.

\section{MATERIAL AND METHODS}

Experimental animals

Thirty healthy male New Zealand white rabbits (average weight: $(2.4 \pm 0.3) \mathrm{kg}$ ) were used in this study. ${ }^{13}$ All experimental procedures were carried out in accordance with the guidelines of the Chinese Ministry of Public Health for the care and use of laboratory animals. The study was approved by the Institutional Ethics Committee of Peking University Health Science Center (IRB 00001052-080-48).

Reagents and antibodies

BTXA was a gift from Lanzhou Institute of Biological Products, China. The titer of 1 U BTXA (Hengli, Lanzhou, China) is equal to that of $1 \mathrm{U}$ BOTOX (Allergan, Irvine, CA, USA) or 3-4 U Dysport (Ipsen, Slough, UK). ${ }^{14}$ Antibodies to M3-muscarinic receptor and AQP5, and horseradish peroxidase-, tetramethylrhodamine isothiocyanate- and fluorescein isothiocyanate (FITC)-conjugated secondary antibodies were purchased from Santa Cruz Biotechnology (Santa Cruz, CA, USA). Other chemicals and reagents were of analytical grade.

Experimental groups

Thirty rabbits were randomly divided into normal control group $(n=5$, without any treatment) and BTXA-treated groups $(n=25)$.

${ }^{1}$ Department of Oral and Maxillofacial Surgery, Peking University School and Hospital of Stomatology, Beijing, China and ${ }^{2}$ Department of Physiology and Pathophysiology, Peking University Health Science Center, Beijing, China

Correspondence: Dr ZG Cai, Department of Oral and Maxillofacial Surgery, Peking University School and Hospital of Stomatology, No. 22 Zhongguanchun South Street, Beijing 100081, China E-mail: czg4209@126.com

Received 10 December 2012; accepted 13 August 2013 
Table 1 Rabbits' primary information of different groups

\begin{tabular}{lcc}
\hline Groups & Weight/kg & Age/week \\
\hline Control & $2.32 \pm 0.15$ & 16 \\
$10 \cup$ & $2.26 \pm 0.15$ & 16 \\
$5 \cup, 1$ Week & $2.28 \pm 0.11$ & 16 \\
$5 \cup, 2$ Week & $2.24 \pm 0.09$ & 16 \\
$5 \cup, 4$ Week & $2.28 \pm 0.08$ & 16 \\
$5 \cup, 12$ Week & $2.32 \pm 0.15$ & 16 \\
\hline
\end{tabular}

The 25 rabbits in BTXA-treated group were further divided in $10 \mathrm{U}$ group ( $n=5$, observed 1 week) and $5 \mathrm{U}$ group ( $n=20$, observed $1,2,4$ and 12 weeks, respectively). Rabbits' primary information of different groups is shown in Table 1 . The animals were anesthetized with sodium pentobarbital $\left(20 \mathrm{mg} \cdot \mathrm{kg}^{-1}\right.$ body weight) for the operation. Local anesthesia was performed by $1 \%$ lidocaine, and glands on both sides were exposed by a median cervical incision. The left SMG was injected with BTXA, while the right SMG was injected with the same volume of normal saline as the contralateral control. At 1, 2, 4 and 12 weeks after operation, a polyethylene tube was inserted into Wharton's duct from the mouth under anesthesia and left outside for measurement and collection of saliva. The glands were then removed under anesthesia, and weighed immediately. Animals were killed with an overdose of barbiturate after removing the glands.

For the injections, BTXA was reconstituted using $2 \mathrm{~mL}$ of $0.9 \%$ saline per $100 \mathrm{U}$, which means that the concentration of BTXA solution was $50 \mathrm{U} \cdot \mathrm{mL}^{-1}$. So the volume of $5 \mathrm{U}$ BTXA is $0.1 \mathrm{~mL}$ and the volume of $10 \mathrm{U}$ BTXA is $0.2 \mathrm{~mL}$.

\section{Measurement of salivary secretion}

Saliva flow was measured for 5 min each at 1,2, 4 and 12 weeks after the operation by the length of filter paper $\left(35 \times 5 \mathrm{~mm}^{2}\right)$ moistened by saliva from the cannula inserted into the Wharton's duct. All the measurements were carried out between 8:00 a.m. and 10:00 a.m., while the rabbits were awake and at rest or during feeding. The collected saliva samples were pooled for biochemical analysis of the concentrations of electrolytes and protein.

\section{Light microscopy and transmission electron microscopy}

The gland sections were stained with hematoxylin and eosin in order to observe the morphological changes by light microscopy. The specimens were fixed in 2\% paraformaldehyde and $1.25 \%$ glutaraldehyde, and then ultrathin sections were stained with uranyl acetate and lead citrate and observed ultrastructure under a transmission electron microscope (JEOL JEM-1400; HITA-CHI, Tokyo, Japan).

\section{Terminal deoxynucleotidyl transferase-mediated deoxyuridine} triphosphate-biotin nick-end labeling staining

Frozen SMG sections were prepared for staining with deoxyuridine triphosphate-biotin nick-end labeling (TUNEL). The in situ cell-death detection kit (Roche Applied Science, Penzberg, Germany) was used according to the manufacturer's instructions. Briefly, tissue sections were incubated with terminal deoxynucleotidyl transferase in a humidified chamber at $37{ }^{\circ} \mathrm{C}$ for $1 \mathrm{~h}$. A mixture of antidigoxigeninperoxidase and substrate-chromagen were used for visualization, and the cells were counterstained with hematoxylin. The nuclei of the apoptotic cells were stained dark brown.

\section{Reverse transcriptase polymerase chain reaction}

Total RNA from SMG was purified using Trizol reagent (Invitrogen, Carlsbad, CA, USA) according to the manufacturer's instructions.
cDNA was prepared from $5 \mu \mathrm{g}$ of total RNA with Moloney murine leukemia virus reverse-transcriptase (Promega, Madison, WI, USA). The sense and antisense primers for M3-muscarinic acetylcholine receptor (M3 receptor) (NM_000740), AQP5 (AF_495879) and $\beta$ actin were $5^{\prime}$-CTT CTC CAA GCT TCC CAT CCA-3' and $5^{\prime}$-TGA CCG ACT GTC TCT GCT GGT-3', 5'-GTT CCT GGC CAC CCT CAT CT- ${ }^{\prime}$ ' and 5' - ACA GAC AGG CCG ATG GAC AG-3' ${ }^{\prime}$, and 5' ATC TGG CAC CAC ACC TTC TAC AAT GAG CTG GCG-3' and 5' -CGC CAT ACT CCT GCT TGC TGA TCC ACA TCT GC-3', respectively. ${ }^{15} \beta$-actin was amplified as the internal standard. The amplification products were visualized on $1.5 \%$ agarose gel with ethidium bromide and sequenced to confirm their identities. The band densities were quantified using a LEICA-550IW image analysis system (Leica, Manheim, Germany).

\section{Immunofluorescence}

Frozen SMG sections were immunostained with anti-AQP5 antibody at a $1: 100$ dilution, and incubated with FITC- or tetramethylrhodamine isothiocyanate-labeled secondary antibodies as described previously. ${ }^{14}$ Nuclei were stained with 4',6 -diamidino-2-phenylindole (Sigma-Aldrich, St Louis, MO, USA). Fluorescence images were captured under a confocal microscope (TCS SP5; Leica, Heidelberg, Germany).

\section{Statistical analysis}

Data are presented as (mean \pm s.d.). Differences among groups were analyzed by one-way analysis of variance, and then Bonferroni testing. A value of $P<0.05$ was considered statistically significant.

\section{RESULTS}

BTXA decreased the secretion of SMGs at rest and after feeding One week after intraglandular injection of BTXA, a statistically significant decrease in rest saliva secretion was observed in the BTXAtreated group at $5 \mathrm{U}$ and $10 \mathrm{U}((1.7 \pm 0.4) \mathrm{mm}$ and $(1.0 \pm 0.3) \mathrm{mm}$ in 5 min, respectively,) compared with the normal control group $((5.4 \pm 1.1) \mathrm{mm}$ in $5 \mathrm{~min}, P<0.01)$. The rest saliva secretion of the glands with $10 \mathrm{U}$ of BTXA was significantly lower than that with $5 \mathrm{U}$ $(P<0.05)$. Dysphagia occurred in three of five rabbits with $10 \mathrm{U}$ of BTXA, while no dysphagia was found in the five rabbits with $5 \mathrm{U}$ of BTXA. To minimize the risk of side effects, we chose $5 \mathrm{U}$ as the optimal dosage for BTXA intraglandular injection to investigate the long-term effects.

There were no differences in rest saliva secretion of the salineinjected SMGs (contralateral control group) and the normal control group $(P>0.05)$. At 1 and 2 weeks after BTXA injection, the volumes of rest saliva in the injected SMGs decreased by $63 \%$ and $70 \%$ of the normal control glands, respectively. At 4 and 12 weeks after BTXA injection, the rest saliva secretion decreased by $54 \%$ and $38 \%$ of the normal control group, respectively, suggesting partial recovery of secretion. During feeding, the stimulated saliva secretion decreased to $69 \%, 70 \%$ and $68 \%$ of the normal control group at 1,2 and 4 weeks after BTXA injection, respectively. The stimulated secretion recovered partly at 12 weeks and showed a decrease of only $41 \%$ of the normal control group (Figure 1a and 1b).

There were no significant differences between the weights of SMGs injected with BTXA and the normal control glands. The weights of SMGs was $(0.49 \pm 0.08) \mathrm{g},(0.55 \pm 0.10) \mathrm{g},(0.56 \pm 0.07) \mathrm{g}$ and $(0.56 \pm$ $0.08) \mathrm{g}$, respectively, in $1,2,4$ and 12 weeks after BTXA injection compared with the normal control group $(0.56 \pm 0.08) \mathrm{g}$. 

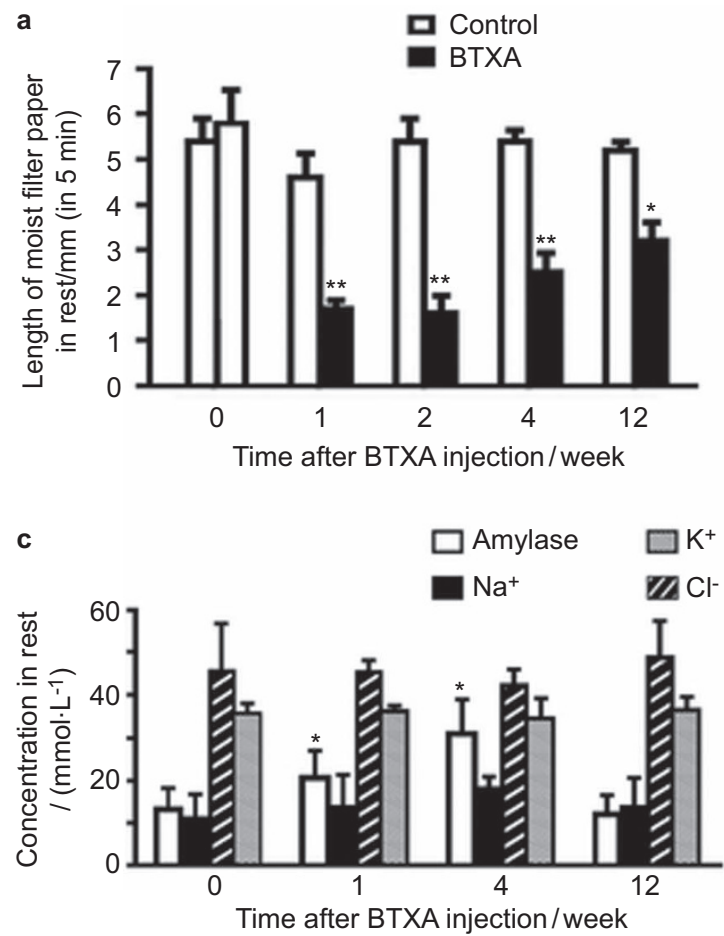


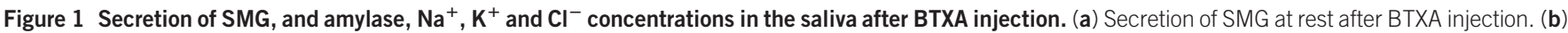

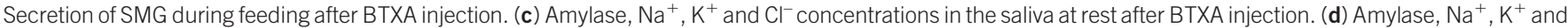
$\mathrm{Cl}^{-}$concentrations in the saliva during feeding after BTXA injection. $* P<0.05, * * P<0.01$ vs. control. BTXA, botulinum toxin type A; SMG, submandibular gland.

Changes in the salivary electrolytes and proteins after BTXA injection

Amylase concentrations in the rest saliva at 1 and 4 weeks after BTXA administration were $55.2 \%$ and $131.3 \%$ higher than those in normal control group; however, potassium, sodium and chlorine concentrations remained unchanged. The sodium concentration in the stimulated saliva collected during feeding at 1 and 4 weeks after BTXA treatment increased to $133.7 \%$ and $159 \%$ of the normal control group $(P<0.05)$; however, the amylase, potassium, and chlorine concentrations did not change (Figure 1c and 1d).

\section{Histological changes after BTXA injection}

Normal acinar and ductal cells could be observed in the normal control SMGs by light microscopy (Figure 2a). No morphological change was found in the contralateral SMGs with normal saline injection. One week after BTXA injection, the acinar and ductal cells in the glandular lobules at the site of BTXA injection was replaced by fibrous tissue. Acinar cells surrounding the injection point showed size reduction, while ducts dilated slightly (Figure $2 \mathrm{~b}$ and $2 \mathrm{c}$ ). Four weeks later, the morphological appearance of BTXA-injected SMGs recovered partly. Twelve weeks later, the treated cells appeared morphologically similar to the normal control glands (Figure $2 \mathrm{~d}$ and $2 \mathrm{e}$ ).

Transmission electron microscopy revealed that secretory granules of low matrix density spread widely in the cytoplasm of acinar cells in the normal control (Figure 3a), while irregular acinar cells were observed in the glands at 1 and 2 weeks after BTXA injection. Secretory granules were abundant in the secretory cells of BTXAinjected glands, and some secretory granules coalesced to form a larger ovoid structure, which resulted in decreased intercellular space (Figure $3 \mathrm{~b}$ and $3 \mathrm{c}$ ). Four weeks later, the morphological appearance of BTXA-injected glands recovered partly. Twelve weeks later, the morphological appearance of the injected glands recovered to that of the normal control glands (Figure $3 \mathrm{~d}$ and $3 \mathrm{e}$ ).

\section{BTXA induced apoptosis in SMGs}

We used TUNEL assays to investigate whether BTXA could induce apoptosis in the SMGs. As shown in Figure 4a, few apoptotic cells were shown in the normal control SMGs. Apoptotic cells appeared in some glandular lobules 1 week after BTXA injection (Figure 4b), and more ductal cells than acinar cells tended to show apoptosis. The apoptotic ductal cells were mainly located in certain gland lobules, and only few apoptotic cells were found in the adjacent lobules. Both acinar and ductal cells showed apoptosis 2 weeks after BTXA injection (Figure 4c). The number of apoptotic cells decreased 4 weeks later (Figure $4 \mathrm{~d}$ ) and almost completely disappeared 12 weeks after BTXA injection (Figure 4e).

\section{BTXA inhibited the expression of M3 receptor}

At 1 and 2 weeks after BTXA injection, the mRNA expression of M3 receptor decreased by $30 \%$ and $50 \%$ of that of the normal control group, respectively, and it began to recover 4 weeks after BTXA injection. mRNA expression of M3 receptor was equal to those of the normal control group at 12 weeks after BTXA injection (Figure 5a).

BTXA inhibited the expression and translocation of AQP5 At 1 and 2 weeks after BTXA injection, the mRNA expression of AQP5 decreased by $26 \%$ and $25.5 \%$ of the normal control group, respectively, in the glands injected with BTXA, and this difference disappeared at 4 weeks after BTXA injection (Figure 5b). Immunofluorescence analysis revealed that AQP5 was predominantly localized in the apical 

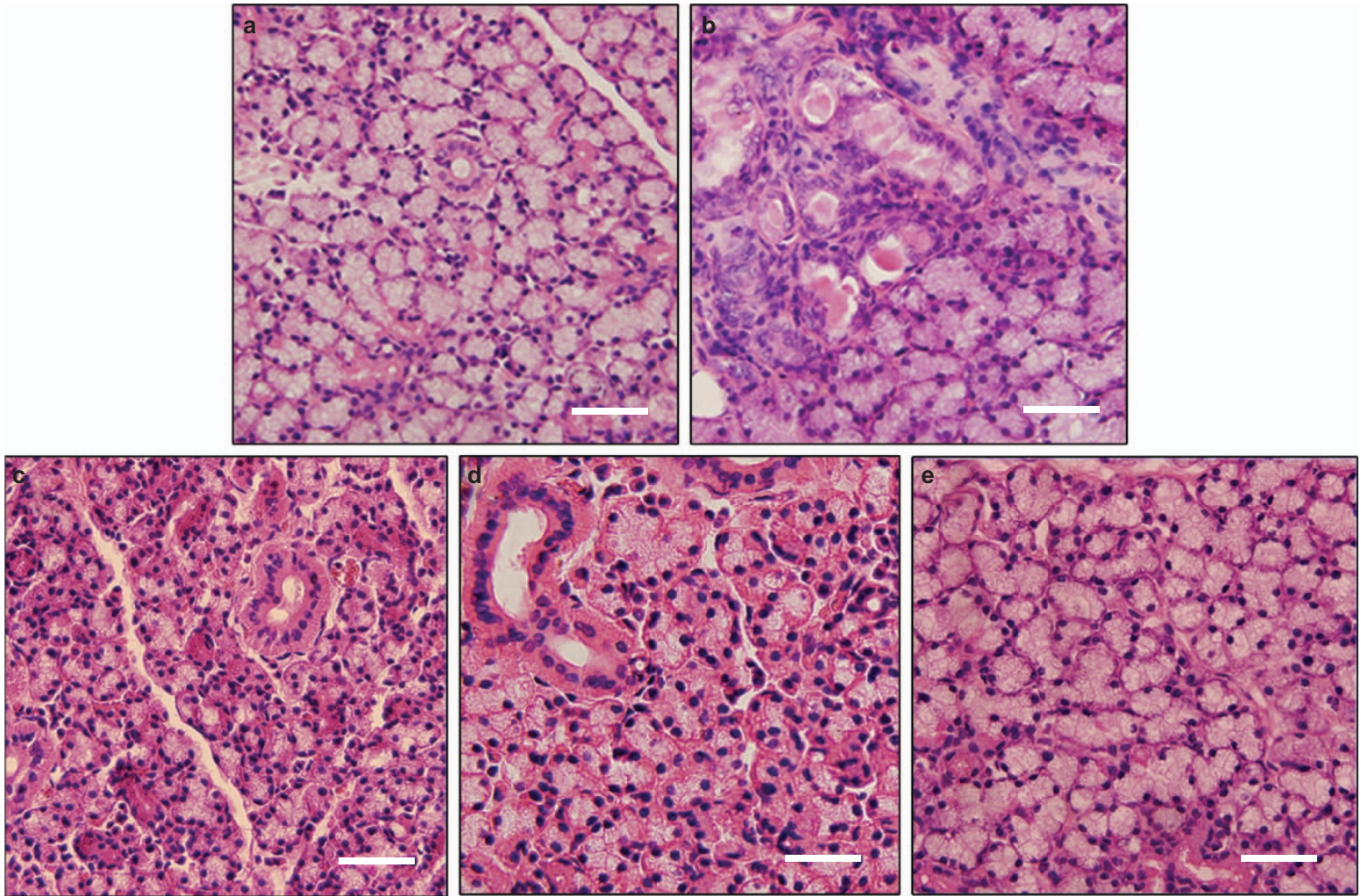

Figure 2 Histological structure of submandibular glands under $\times 400$ magnification. (a) Histological evaluation of a control submandibular gland. (b) Submandubular gland at 1 week after BTXA injection. The normal structure of acinar and ductal cells in the gland lobule at the site of the injection disappeared and was replaced by fibrous tissue. (c) Submandubular gland at 2 weeks after BTXA injection. Acinar cells showed a reduction in size, while the ductal cells expanded slightly. (d) Submandubular gland at 4 weeks after BTXA injection. (e) Submandubular gland at 12 weeks after BTXA injection. The morphological appearance of acinar cells recovered to the normal. Bar $=50 \mu \mathrm{m}$. BTXA, botulinum toxin type $\mathrm{A}$.
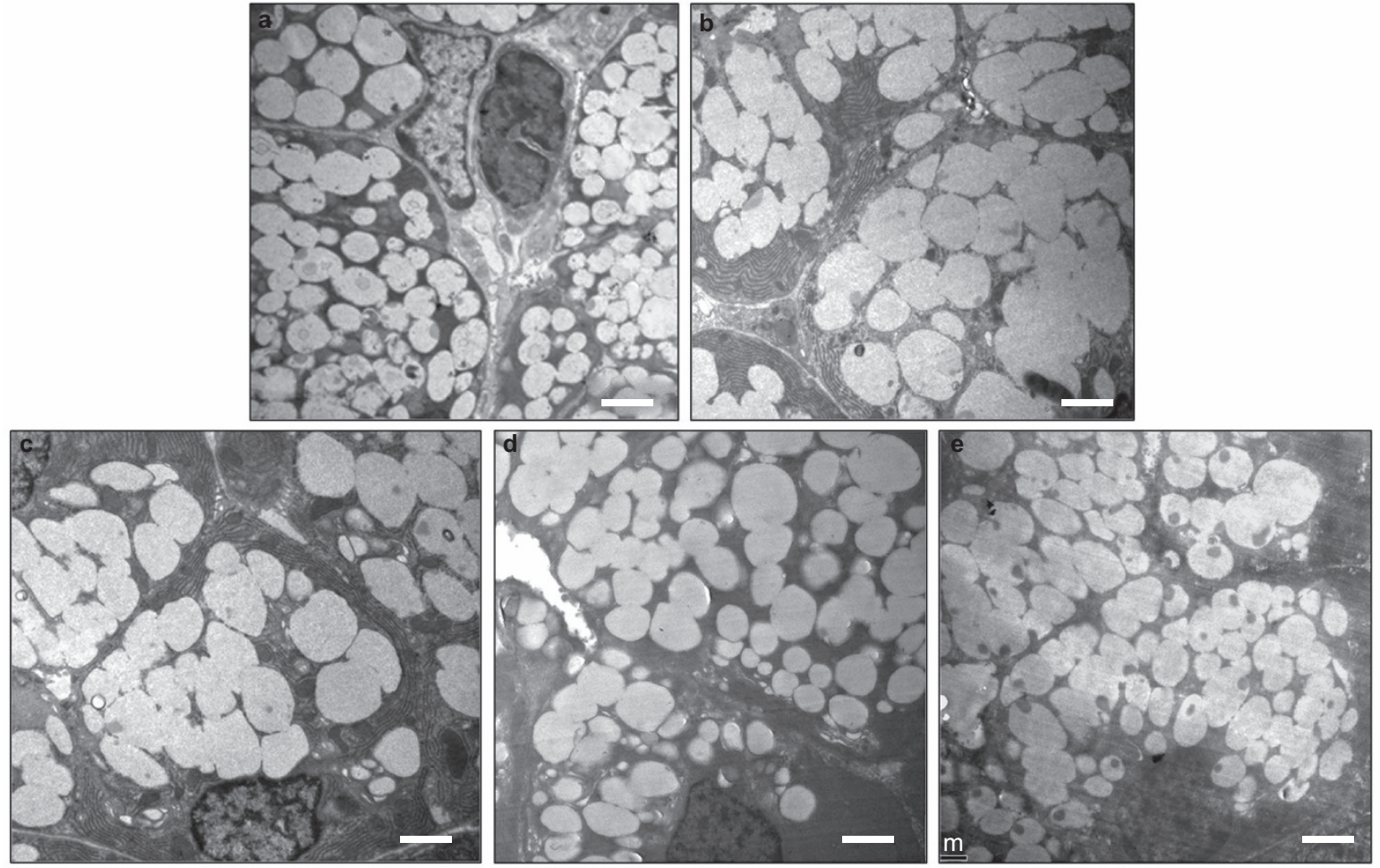

Figure 3 Ultrastructure of submandibular gland under $\times 5000$ magnification. Ultrastructures of (a) control, (b) 1 week after BTXA injection, (c) 2 weeks after BTXA injection, (d) 4 weeks after BTXA injection and (e) 12 weeks after BTXA injection. Bar $=2 \mu \mathrm{m}$. BTXA, botulinum toxin type A. 

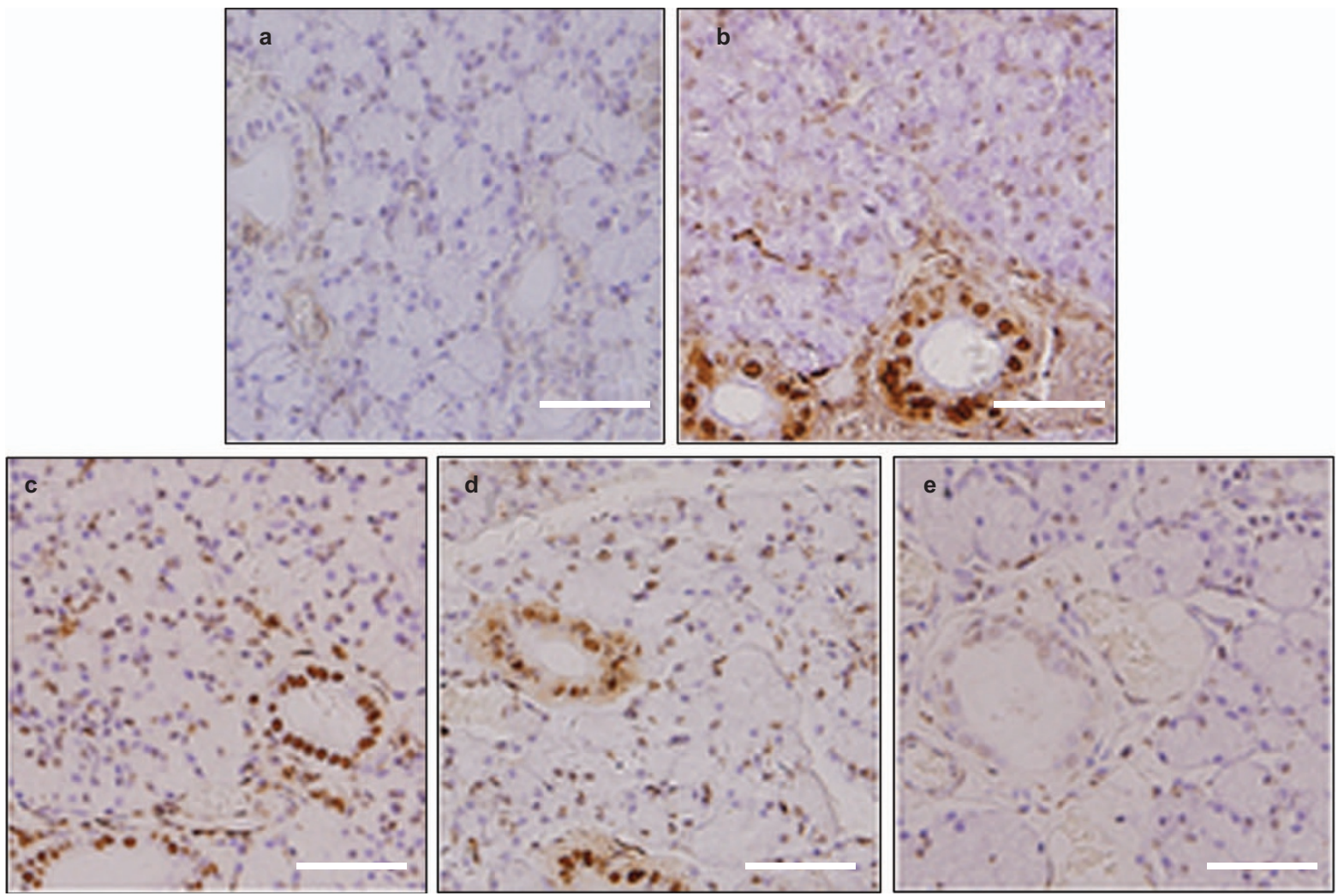

Figure 4 TUNEL staining in SMG. (a) Very few cells were stained in the control SMG. (b) One week after BTXA injection, numerous typical densely stained dark TUNEL-positive cell nuclei can be observed in ductal cells. (c) Two weeks after BTXA injection, apoptotic cells can be seen in the acinar and ductal cells. (d) TUNEL-positive cells decreased at 4 weeks after BTXA injection. (e) TUNEL-positive cells almost disappeared in the glands at 12 weeks after BTXA injection. Magnification $\times 400$. Bar=50 $\mu \mathrm{m}$. BTXA, botulinum toxin type A; SMG, submandibular gland; TUNEL, terminal deoxynucleotidyl transferase-mediated deoxyuridine triphosphate-biotin nick-end labeling.
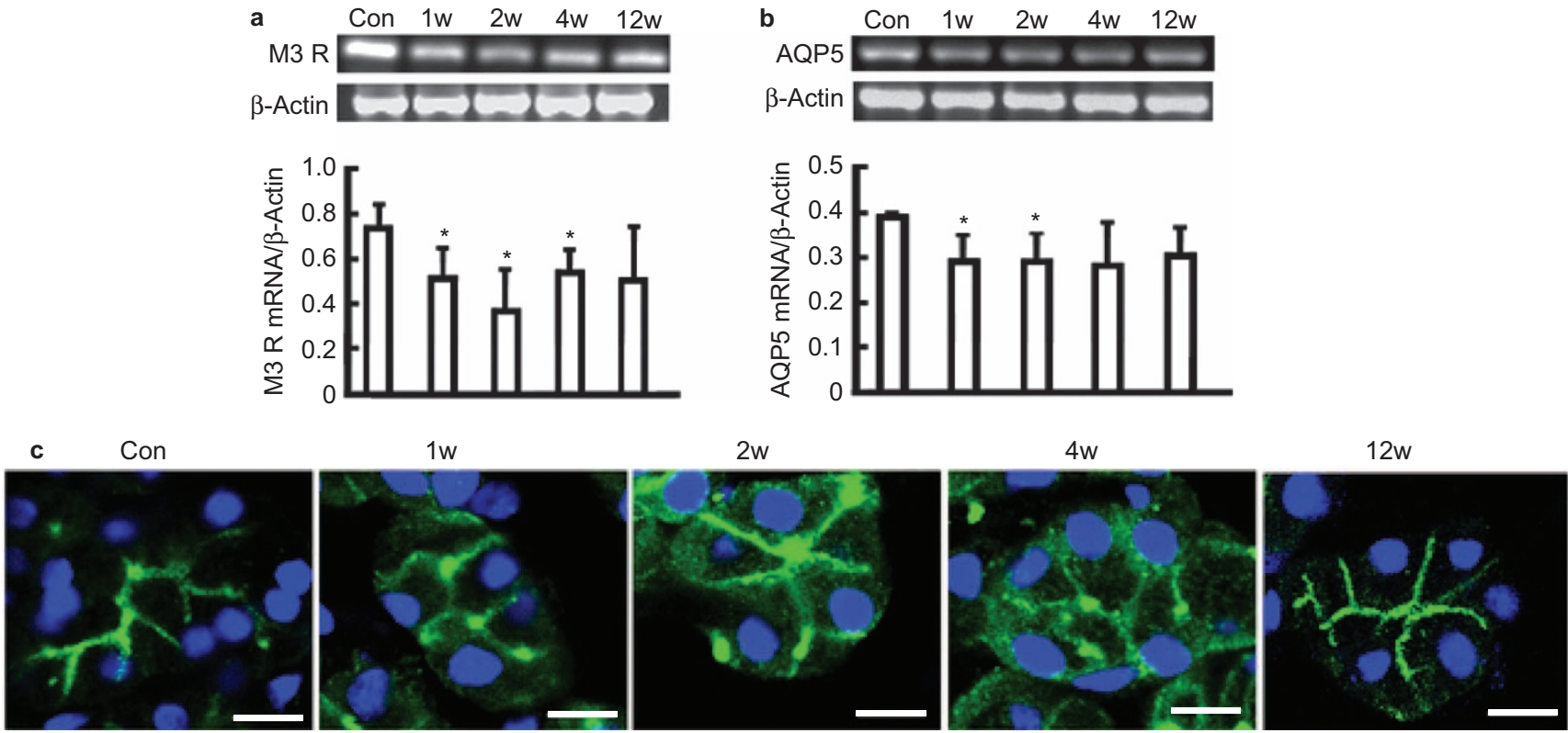

Figure 5 The expression of M3 receptor and AQP5. The mRNA expression of M3 receptor (a) and AQP5 (b) were detected by RT-PCR. (c) Effect of BTXA on the distribution of AQP5 in submandibular glands. Representative immunofluorescence images of AQP5 in the control gland, at 1,2, 4 and 12 weeks after BTXA injection. Magnification $\times 1$ 200. Bar $=10 \mu \mathrm{m}$. Con, control; w, week; M3 R, M3 receptor, M3-muscarinic acetylcholine receptor; AQP5, aquaporin-5; BTXA, botulinum toxin type A; RT-PCR, reverse transcriptase polymerase chain reaction; SMG, submandibular gland. 
membranes of acinar cells in the normal control glands, while in BTXAinjected glands, AQP5 expression was diminished in the apical membranes and dispersed in the cytoplasm. AQP5 relocalized in the apical membranes of acinar cells at 12 weeks after BTXA injection (Figure 5c).

\section{DISCUSSION}

Drooling caused by cerebral palsy and Parkinson's disease may be disturbing, resulting in chronic irritation of the facial skin. ${ }^{16}$ Other symptoms related to salivary secretion, such as salivary fistula and epiphora after autologous SMG transplantation, also lead to decline of quality of life and social embarrassment. ${ }^{17}$ Anticholinergic medication, surgery and other therapy have all been used to reduce excessive secretion with various side effects. It is critical to find a safe and effective therapy to treat these symptoms.

BTXA has been used to treat drooling with encouraging results. ${ }^{5-7}$ Our study revealed that injection of 5 units BTXA in the SMG decreased saliva secretion in rabbits significantly at rest and during feeding without side effects, such as dysphagia, and the secretary function partly recovered 4 weeks after the injection. The salivary secretion remained lower at 12 weeks after the BTXA injection than that in normal controls, indicating that BTXA has a longer but reversible effect on the secretion of salivary gland. It may be applied in the treatment of symptoms related to salivary secretion such as drooling and epiphora after autologous SMG transplantation.

It is noticeable that the compositions of saliva change after BTXA treatment. The primary saliva secreted from acinar cells is a plasmalike fluid containing high $\mathrm{Na}^{+}$and low $\mathrm{K}^{+}$concentrations. When the saliva flows through the ductal system, its composition is greatly modified by the ductal cells through re-absorption of $\mathrm{Na}^{+}$and $\mathrm{Cl}^{-}$ and release of $\mathrm{K}^{+}$. Stimulation of the cholinergic receptors activates these ion transporters in ductal cells. ${ }^{18}$ Our study showed increased amylase concentrations in the rest and stimulated saliva after BTXA treatment, while the $\mathrm{K}^{+}$and $\mathrm{Cl}^{-}$concentrations did not change, which may be related to a lack of acetylcholine by BTXA, causing decreased fluid and ion secretion. Thus, the decreased fluid secretion and unaltered protein secretion leads to the resultant high protein concentration observed in the saliva.

It has been reported that BTXA treatment induced a decrease in the glandular weight and reduction in the size of acinar cells in the rats. ${ }^{19-20}$ The lumen of the granular striated ducts was found to be wider following BTXA injection than that in the controls. ${ }^{21}$ In our study, at 1 week after BTXA injection, destruction of the gland lobule with dilated duct and hyperplastic connective tissue was observed at the injection site. Acinar cells of the SMG lobules near the injection site shrunk dramatically, while the acinar cells located at a distance from the injection site showed only slight change in size. Ultrastructure studies revealed old secretory granules in acinar cells coalesced to form larger ovoid structures. Results of the histological analysis revealed that the BTXA affected mainly those acinar cells located around the site of injection, and decreased the secretory function of these cells, but did not destroy the acinar lobules away from the injection site.

The mechanism of decreased saliva secretion by BTXA treatment is not clear. The release of acetylcholine from parasympathetic nerves and its interaction with muscarinic acetylcholine receptors regulates glandular fluid and ion secretion. ${ }^{22-23}$ Our study showed that the expression of M3 receptor decreased at 1 week after BTXA injection and began to recover at 4 weeks after the injection, and the levels returned to $80 \%$ of the control at 12 weeks after the injection. It indicated that BTXA-induced decreased salivary secretion is related to decreased expression of $\mathrm{M} 3$ receptor.

BTXA can induce apoptosis in nasal glands of guinea pigs, and apoptotic cells have been found up to three months after treatment with BTXA. ${ }^{24-25}$ Intraprostatic injection of BTXA has been shown to induce prostate apoptosis in dogs. ${ }^{26}$ In our study, apoptosis of the SMG cells occurred 1 week after BTXA treatment, reached to the maximum level 2 weeks after BTXA treatment, and the apoptotic cells almost disappeared 12 weeks after the BTXA treatment. The change in the number of apoptotic cells was parallel with changes in the secretion of the SMG after BTXA injection, suggesting that the decreased salivary secretion might be related to cell apoptosis.

AQP5 plays a major role in salivary fluid secretion. Localization of AQP5 is critical for regulating salivary fluid secretion. ${ }^{12,27}$ Interestingly, our study demonstrated that deactivation of M3 receptor by BTXA decreased mRNA expression of AQP5 and prevented its translocation from the cytoplasm to the apical membrane. This new finding suggests that the decreased saliva secretion caused by BTXA may be mediated by the inhibition of AQP5 expression and trafficking.

In summary, our study found that BTXA injection could reversibly decrease the saliva secretion from SMGs. BTXA might be effectively used to treat symptoms related to salivary secretion such as drooling and epiphora after autologous SMG transplantation. BTXA injection decreased expression of M3 receptor and AQP5, inhibited redistribution of AQP5 and induced cell apoptosis in SMG. It indicates that mechanism of BTXA-mediated decreased salivary secretion might be related to change of M3 receptor signaling pathway and cell apoptosis.

\section{ACKNOWLEDGEMENTS}

This study was supported by grants from the National Natural Science Foundation of China (Nos. 30973336 and 81100762).

1 Quagliato EM, Carelli Ef, Viana MA. A prospective, randomized, double-blind study comparing the efficacy and safety of type a botulinum toxins botox and prosigne in the treatment of cervical dystonia. Clin Neuropharmacol 2010; 33(1): 22-26.

2 Mezali T, Kaji R, Brin MF et al. Combined use of type A and F botulinum toxins for blepharospasm: a double-blind controlled trial. Mov Disord 1999; 14(6): 1017-1020.

3 Krause E, Schirra J, Gürkov R. Botulinum toxin a treatment of cricopharyngeal dysphagia after subarachnoid hemorrhage. Dysphagia 2008; 23(4): 406-410.

4 Rieder CR, Schestatsky P, Socal MP et al. A double-blind, randomized, crossover study of prosigne versus botox in patients with blepharospasm and hemifacial spasm. Clin Neuropharmacol 2007; 30(1): 39-42.

5 Hassin BS, Scheuer E, Buchman AS et al. Botulinum toxin injections for children with excessive drooling. J Child Neurol 2005; 20(2): 120-123.

6 Gabreels FJ, Hulst KV, Rotteveel JJ et al. Effect of botulinum toxin in the treatment of drooling: a controlled clinical trial. Pediatrics 2004; 114(3): 620-627.

7 Lin YC, Shieh JY, Cheng ML et al. Botulinum toxin type A for control of drooling in Asian patients with cerebral palsy. Neurology 2008; 70(4): 316-320.

8 Ellies M, Laskawi R, Gotz W et al. Immunohistochemical and morphometric investigations of the influence of botulinum toxin on the submandibular gland of the rat. Eur Arch Otorhinolaryngol 1999; 256(3): 148-152.

9 Melvin JE, Yule D, Shuttleworth T et al. Regulation of fluid and electrolyte secretion in salivary gland acinar cells. Annu Rev Physiol 2005; 67: 445-469.

10 Baum BJ, Wellner RB. Neural mechanisms of salivary gland secretion. Basel: Karger, 1999.

11 Baum BJ. Principles of saliva secretion. Ann NY Acad Sci 1993; 694: 17-23.

12 Ishikawa Y, Eguchi T, Skowronski MT et al. Acetylcholine acts on M3 muscarinic receptors and induces the translocation of aquaporin5 water channel via cytosolic $\mathrm{Ca}^{2+}$ elevation in rat parotid glands. Biochem Biophys Res Commun 1998; 245(3): 835-840.

13 Xiang B, Zhang Y, Li YM et al. Effects of phenylephrine on transplanted submandibular gland. J Dent Res 2006; 85(12): 1106-1111.

14 Wang YC, Han YZ. [Application of botulinum toxin A in aesthetic medicine]. Chin $J$ Aesth Med 2002; 11(1): 88-91. Chinese.

15 Shi L, Zhang Y, Ding C et al. Carbachol improves secretion in the early phase after rabbit submandibular gland transplantation. Oral Dis 2010; 16(4): 351-359. 
16 Reid SM, Johnstone BR, Westbury CR et al. Randomized trial of botulinum toxin injections into the salivary glands to reduce drooling in children with neurological disorders. Dev Med Child Neurol 2008; 50(2): 123-128.

17 Geerling G, Garrett JR, Paterson KL et al. Innervation and secretory function of transplanted human submandibular salivary glands. Transplantation 2008; 85(1): 135-140.

18 Turner RJ, Sugiya H. Understanding salivary fluid and protein secretion. Oral Dis 2002; 8(1): 3-11.

19 Wen WD, Yuan F, Hou YP. [The mechanism of inhibitory effect on parotid gland secretion with local injection of botulinum toxin type A in the rat.] Zhonghua Kou Qiang Yi Xue Za Zhi 2009; 44(1): 38-40. Chinese.

20 Coskun BU, Savk H, Cicek ED et al. Histopathological and radiological investigations of the influence of botulinum toxin on the submandibular gland of the rat. Eur Arch Otorhinolaryngol 2007; 264(7): 783-787.

21 Teymoortash A, Sommer F, Mandic R et al. Intraglandular application of botulinum toxin leads to structural and functional changes in rat acinar cells. Br J Pharmaco 2007; 152(1): 161-167.

22 Proctor GB. Muscarinic receptors and salivary secretion. J App/ Physio/2006; 100(4): 1103-1104.
23 Nakamura T, Mastsui M, Uchida K et al. M3 muscarinic acetylcholine receptor plays a critical role in parasympathetic control of salivation in mice. J Physiol 2004; 558(2): 561-575.

24 Lim M, Mace A, Nouraei SA et al. Botulinum toxin in the management of sialorrhoea: a systematic review. Clin Otolaryngol 2006; 31(4): 267-272.

25 Olthoff A, Laskawi R, Gieffer B et al. Botulinum toxin type A induced apoptosis in nasal glands of guinea pigs. Ann Otol Rhinol Laryngol 2001; 110(11): 1045-1050.

26 Chuang YC, Tu CH, Huang CC et al. Intraprostatic injection of botulinum toxin type-A relieves bladder outlet obstruction in human and induces prostate apoptosis in dogs. BMC Urol 2006; 6: 12.

$27 \mathrm{Ma} \mathrm{TH}$, Song $\mathrm{YL}$, Gillespie A et al. Defective secretion of saliva in transgenic mice lacking aquaporin-5 water channels. J Biol Chem 1999; 274(29): 20071-20074.

(c)

This work is licensed under a Creative Commons

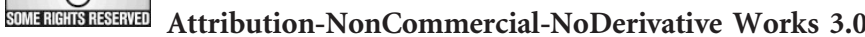
Unported License. To view a copy of this license, visit http:// creativecommons.org/licenses/by-nc-nd/3.0 